\title{
Synthesis of Nanoparticles an Overew, a Review Article
}

\author{
S. D. Jadhav', I A Shaikh² \\ ${ }^{1}$ Associate Professor, ${ }^{2}$ Assistant Professor \\ ${ }_{1,2}$ Department of Basic Science \& Humanities, Bharati Vidyapeeth, \\ 1,2(Deemed to be University), College of Engineering, Pune, Maharashtra, India
}

\begin{abstract}
How to cite this paper: S. D. Jadhav | I A
Shaikh "Synthesis of Nanoparticles an Overew, a Review Article" Published in International Journal of Trend in Scientific Research and Development (ijtsrd), ISSN: 24566470, Volume-3 | Issue-3, April 2019, pp.426-428, URL: http://www.ijtsrd.co $\mathrm{m} /$ papers/ijtsrd228 16.pdf

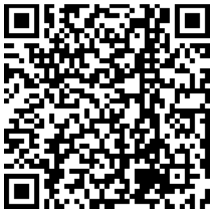
IITSRD22816
\end{abstract}

ABSTRACT

Nanotechnology is an interdisciplinary subject. A large number of techniques like physical, mechanical, chemical, biological and hybrid are available to synthesize different types of nanomatericals. Synthesized nanoparticles are in the form of colloids, clusters, powders, tubes, rods, wires and thin films etc. The technique to be used depends upon the material of interest, type of nanomaterial, size and quantity. A full potential of this technology can be exploited for the advantage of mankind. However, to fully exploire the potential of nanotechnology, it is essential to know what are nanomaterials, how these materials can be synthesized. An attempt is made for this by giving some of techniques used to synthesize nanomaterials by this review article.

Copyright (C) 2019 by author(s) and International Journal of Trend in Scientific Research and Development Journal. This is an Open Access article distributed under the terms of the Creative Commons

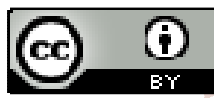

Attribution License (CC BY 4.0) (http://creativecommons.org/licenses/ by/4.0)

\section{INTRODUCTION:}

Nanoparticles can be classified into various types, according to their size, shape, and material properties. Some classifications distinguish between organic and inorganic nanoparticles, the way in which nanoparticles are classified typically depends on their applications, or may be related to the way in which they were produced. Nanoparticles exist in the natural world and are also created as a result of human activities. Because of their submicroscopic size, they have unique material characteristics, and manufactured nanoparticles may find practical applications in a variety of areas. International Organization for Standardization (ISO) defined a nanoparticle as a discrete nano-object where all three Cartesian dimensions are less than $100 \mathrm{~nm}$. The ISO standard similarly defined two-dimensional nano-objects and one-dimensional nano-objects. But later on the definition is modified. In addition, nanoparticles can be classified as hard, silica particles and fullerenes or as soft nanodroplets.

The study of biological systems and the engineering of many materials such as colloidal dispersions, metallic quantum dots, and catalysts have been in the nanometer regime for centuries. For example, the Chinese are known to use $\mathrm{Au}$ nanoparticles as an inorganic dye to introduce red color into their ceramic porcelains more than thousand years ago. Use of colloidal gold has a long history, though a comprehensive study on the preparation and properties of colloidal gold was first published in the middle of the 19th century. Colloidal dispersion of gold prepared by Faraday in 1857 was stable for almost a century before being destroyed during World War II. Medical applications of colloidal gold present another example. Colloidal gold was, and is still, used for treatment of arthritis.

In general, nanotechnology can be understood as a technology of design, fabrication and applications of nanostructures and nanomaterials. Nanotechnology also includes fundamental understanding of physical properties and phenomena of nanomaterials and nanostructures. Study on fundamental relationships between physical properties and phenomena and material dimensions in the nanometer scale, is also referred to as nanoscience. In the United States, nanotechnology has been defined as being concerned with materials and systems whose structures and components exhibit novel and significantly improved physical, chemical and biological properties, phenomena and processes due to their nanoscale size. Some of the methods used for synthesize nanomaterials are discussed here.

\section{SYNTHESIS OF NANOPARTICLES}

Mechanical Methods

\section{A. High Energy Ball Milling:}

This is one of the simplest ways of making nanoparticles of some metals and alloys in the powder form. Various types of mills can be used in this process. To make large quantities of fine particles, usually one or two containers are used. Size of 
the container depends on size of nanoparticles. Hard steel balls are kept in container along with powder of a material of interest. Container is closed with lids, generally 2:1 mass ratio of balls to material is sufficient. Heavy milling balls increase the impact energy on collision. Larger balls used for milling, produce smaller grain size but having more defects in the particles. The process may add some impurities from balls. Rise in temperature range from 100 to $1100^{\circ} \mathrm{C}$ is expected during collisions. Lower temperature leads to amorphous particle formation. During the milling liquids also can be used. The containers are rotated at high speed ( a few hundred rpm) around their own axis. Further they may rotate around some central axis. At this stage the material is forced to the walls and is pressed against the walls. But due to the motion of the containers around their own axis the material is forced to other region of the container. By using this method material like $\mathrm{Co}, \mathrm{Cr}, \mathrm{W}, \mathrm{Ni}-\mathrm{Ti}, \mathrm{Al}-\mathrm{Fe}$ etc. are converted to nanoparticles.

\section{B. Melt Mixing:}

Nanoparticles can be synthesized in the glass by this method. As we are well known that glass is an amorphous solid, due to symmetrical arrangement of atoms or molecules. When the liquid is cooled below certain temperature (Tm) it forms either a crystalline or amorphous solid i.e. glass. Apart from temperature, the rate of cooling and nucleation formation tendency decides whether the melt can be cooled as a glass or crystalline solid with long range order. Nuclei are formed spontaneously with homogeneous or inhomogeneous nucleation which can grow to form crystalline solids, but if cooled at very high cooling rate $\left(\sim 10^{5}-10^{6} \mathrm{~K} / \mathrm{S}\right)$ they can form amorphous solids. Such solids are called as metallic glasses. Addition of elements like B, P, Si etc. can help the metallic glasses in amorphous state. Hence, it is possible to form nanocrystals within metallic glasses by controlled heating. It is possible to form some of nanoparticles by mixing the molten streams of metals at high velocity with turbulence. On mixing thoroughly, nanoparticles are formed. Nanoparticles of $\mathrm{TiB}_{2}$ can be formed by this method. [1-3]

\section{Methods Based on Evaporation}

A. Physical Vapour Deposition:

This method usually involves use of materials of interest as sources of evaporation. An inert gas or reactive gas for collisions with material vapour. A cold finger on which nanoparticles can condense, a scraper to scrape nanoparticles and piston-anvil. All the processes are carried out in a vaccum chamber so that the desired purity of the end product can be obtained. Generally, high vapour pressure metal oxides are evaporated from filaments of refractory metals like $\mathrm{W}, \mathrm{Ta}$, Mo in which the materials to be evaporated are held. The density of the evaporated material close to the source is quite high and particle size is small $(\leqslant$ $5 \mathrm{~nm}$ ) such particles would prefer to acquire a stable lower energy state. Due to small particle -particle interaction bigger particles can be formed. Hence, they should be removed away from the source as fast as possible. This is done by forcing an inert gas near the source, which removes the particles from the vicinity of the source. In general, the rate of evaporation and the pressure of gases inside the chamber determine the particle size. Evaporated atoms and clusters tend to colloid with gas molecules and make bigger particles, which condense on cold finger. While moving away from cold finger the clusters grow. If clusters have been formed on inert gas molecules or atoms, on reaching the cold finger, gas atoms may leave the particles there and then escape to the gas phase. If the reactive gases like $\mathrm{O}_{2}, \mathrm{~N}_{2}, \mathrm{H}_{2}$ and $\mathrm{NH}_{3}$ etc. are used in the system, evaporated material can interact with these gases forming oxide, nitride, or hydride particles. Size, shape of the evaporated material can depend upon the gas pressure in deposition chamber. Using gas pressure of $\mathrm{H}_{2}$ more than $500 \mathrm{~K} \mathrm{~Pa}$. TiH 2 particles of $\sim 12 \mathrm{~nm}$ size can be produced.

\section{B. Laser Ablation:}

In this method, vapourization of the material is effected using pulses of laser beam of high power. The setup is high vaccum system equipped with inert gas introduction facility, laser beam. Clusters of any material of which solid target can be made are possible to synthesize. Laser which gives UV wavelength such as excimer laser is required as other wavelengths like IR or visible are often are reflected by some of the metal surfaces. A powerful beam of laser evaporates the atoms from a solid source and atoms colloide with inert gas atoms and cool on them forming clusters. They condense on the cooled substrate. This method is known as laser ablation. Single wall Carbon Nanotubes (SWNT) is mostly synthesized by this method [6-7].

\section{Chemical Methods}

\section{A. Colloids synthesis:}

These are the phase separated sub micrometer particles in the form of spherical particles, rods, tubes and plates etc. Theese are the particles suspended in some hot matrix. Metal, alloy, semiconductor and insulator particles of different sizes and shapes can be synthesized in aqueous or non-aqueous medium. Synthesis of colloids is a very old method. M. Faraday synthesized gold nanoparticles by wet chemical route. The particles are so stable. Colloidal particles are synthesized in a glass reactor. Glass reactor has a provision to introduce some precursors, gases as well as measure temperature, $\mathrm{pH}$ etc; during the reaction. It is possible to remove the products at suitable time intervals. Reaction is carried out under inert atmosphere so as to avoid any uncontrolled oxidation of the products. [8]

\section{B. Synthesis of Metal Nanoparticles by Colloidal Method:}

This process is done by reduction of some metal salt or acid. For example copper particles can be obtained by reducing Chloroauric acid $\left(\mathrm{HAuCl}_{4}\right)$ with tri sodium citrate $\left(\mathrm{Na}_{3} \mathrm{C}_{6} \mathrm{H}_{5} \mathrm{O}_{7}\right)$. The reaction will be,

$\mathrm{HAuCl}_{4}+\mathrm{Na}_{3} \mathrm{C}_{6} \mathrm{H}_{5} \mathrm{O}_{7} \rightarrow \mathrm{Au}^{+}+\mathrm{C}_{6} \mathrm{H}_{5} \mathrm{O}_{7^{-}}+\mathrm{HCl}+3 \mathrm{NaCl}$

The reaction will be carried out in water. Obtained nanoparticles exhibit colour depending upon the particle size. i.e. (intense red colour for gold metal). In similar way Silver, Gold, Palladium and few other metal nanoparticles can be synthesized using appropriate precursors, temperature, $\mathrm{pH}$, duration of synthesis etc. [4-5]

\section{Sol-Gel Method:}

In this method two types of materials or compounds 'sol ' and 'gel' involves. This process is a low temperature process, hence less energy consumption and less pollution. Sols are solid particles in a liquid. They are a sub class of colloids. Gels are nothing but a continuous network of particles with pores filled with liquid. A sol gel process involves formation of sols in a liquid and then connecting the sol particles to form a network. By drying the liquid, it is possible to obtain 
powders and thin films. This method is useful to synthesize ceramics or metal oxides, sulphides, borides and nitrides. Sol-gel synthesis involves hydrolysis of precursors, condensation followed by polycondensation to form particles, gelation and drying process by various routes. Precursors are to be chosen so that they have a tendency to form gels. Both alkoxides and metal salts can be used. It is also possible to synthesize nanoparticles like nanorods, nanotubes etc. by sol-gel technique.[14]

\section{Biological Methods}

\section{A. Synthesis using Plant Extracts:}

Use of plants in synthesis of nanoparticles is quite less studied area as compared to use of micro-organisms to produce nanoparticles. There are few examples which suggest that plant extracts can be used in synthesis of nanoparticles. To obtain gold nanoparticles from geranium plant extract is discussed here. Finely crushed leaves are put in Erlenmeyer flask and boiled in water just for a minute. Leaves get ruptured and cells release intracellular material. Solution is cooled and decanted. This solution is added to $\mathrm{HAuCl}_{4}$ aqueous solution, and nanoparticles of gold starts forming with a minutes.[9]

\section{B. Synthesis using DNA:}

CdS nanoparticles can be synthesized by DNA. DNA is used to bind the surface of growing nanoparticles. For example, double standard Salmon Sprem DNA can be sheared to an average size of $500 \mathrm{bp}$. Cadmium acetate can be added to desired medium like water, ethanol, propanol, etc. and reaction is carried out in a glass flask with facility to purge the solution and flow with an inert gas like nitrogen. Addition of DNA should be made and then $\mathrm{Na}_{2} \mathrm{~S}$ can be added drop wise. Depending upon the concentrations of cadmium acetate, sodium chloride DNA nanoparticles of $\mathrm{CdS}$ with sizes less than $\sim 10 \mathrm{~nm}$ can be obtained. It is found that $\mathrm{CdS}$ nanoparticles synthesized by this method have cadmium rich surface. DNA probably bends through its negatively charged phosphate group to positively charged $\left(\mathrm{Cd}^{+}\right)$ nanoparticles surface. The other end of DNA is in fact free to interact with suitable proteins. Nanoparticles prepared by this way are used as sensors of proteins. $[10,15]$

\section{APPLICATIONS}

Due to some of the properties of nanoparticles and size of nanoparticles, they are used in composite materials and as an inorganic filler such as carbon black or silica nanoparticles and nanocomposites. Nanocomposites were employed in the development and design of new materials. As an example, it can be used as the building blocks for new dielectric insulating and magnetic materials. In case of polymers to improve their strength and impact resistance. Nanoparticles have been increasingly incorporated into food packaging to control the ambient atmosphere around food, keeping it fresh and safe from microbial contamination. Such composites use nanoflakes of clays and claylike particles, which slow down the ingress of moisture and reduce gas transport across the packaging film. Nanoparticles that exhibit antimicrobial activity had also been incorporated into paints and coatings, making those products particularly useful for surfaces in hospitals and other medical facilities and in areas of food preparation. Composite materials based on carbon nanotubes and layered-type materials, such as graphene also observed in many places. Nanoparticles and nanofibres plays an important part in the design and manufacture of novel scaffold structures for tissue and bone repair, and many more applications in medicine. [11-13]

\section{CONCLUSION}

Due to small size of the nanoparticles, as compared to microparticles, these are widely used in all fields of research as well as in medical sciences now a day.

\section{REFERENCES}

[1] Nikam A.P: Mukesh P; R ChaudharyS.P; "Nanoparticlesan overew ", International Journal of Research and Development in Pharmacy and Life Sciences. Vol.3 No.Pages1121-1127, 2014.

[2] Wang, D., Xie, T. and Li, Y. (2009) 'Nanocrystals: Solution-Based Synthesis and Applications as Nanocatalysts', Nano Research, Vol. 2, pp.30-46.

[3] Virji, S., Kojima, R., Fowler, J.D., Villanueva, J.G., Kaner, R.B. and Weiller, B.H. (2009) 'Polyaniline Nanofiber Composites with Amines: Novel Materials for Phosgene Detection', Nano Research, Vol. 2, No. 2, pp.135-142.

[4] Y. Liu, F. Yang and X. Yang, Colloids and Surfaces A. 312, (2008), 219

[5] S. V. Manorama, C. V. Gopal Reddy and V. J. Rao, Nanostruct. Mater. 11, (1999), 643.

[6] J. Kong, A.M. Cassel and H.J. Dai, "Chemical vapor deposition of methane for single wallnanotube", Chem.P hys. Lett. Vol. 292, pp. 567-574, 1998.

[7] T.W. Ebbesen, "Synthesis of nanostructured carbon using CVD", Phys. Today, Vol. 49, pp. 26-32, 1996.

[8] R. Srivastava, Synthesis and characterization techniques of nanomaterials, International Journal of Green Nanotechnology: Physics and Chemistry, Vol. 4, pp. 1-11, 2012.

[9] B.C. Yadav, R. Kumar, R. Srivastava and T. shukla, Flame Synthesis of Carbon Nanotubes using Camphor and its Characterization, International Journal of Green Nanotechnology: Physics and Chemistry, Vol. 3, pp. 170-179, 2011.

[10] R. Saito, M. Fujita, G. Dresselhaus, and M. S Dresselhaus "Electronic structure of chiralgraphenetubules", Appl. Phys. Lett. Vol. 60, pp. 2204-2206, May 1992.

[11] Allhoff, F., P.Lin, D.Moore, "The basics of nanotechnology".In: What Is Nanotechnology and Why Does It Matter? From Science to Ethics. New York, NY: John Wiley; 2010: 1-19.

[12] Agrawal, S., R. Prajapati, "Nanosensors and their pharmazeutical applications: a review", MS ID: IJPSN11-20-11-AGRAWAL, Int. J. Pharm. Sci. Nanotech. 4, 4 (2012).

[13] P. Milani and S. Iannota, "Cluster beam synthesis of nanostructured materials", Springer Verlag, Berlin Heidelberg, New York (1999).

[14] R.W. Jones, "Fundamental principles of sol-gel technology", The Institute of Metals (1989).

[15] Absar Ahmad, Murali Sastry, M. Islam Khan and Rajiv Kumar, "Biosynthesis of metal nanoparticles using fungi and actinomycete, Curr. Sci. 85: 162 (2003). 